\title{
Factors determining the nutrition transition in two Mediterranean islands: Sardinia and Malta
}

\author{
Sophie Tessier* and Mariette Gerber \\ Groupe d'Epidémiologie Métabolique, Centre de Recherche en Cancérologie, INSERM-CRLC, F-34298 Montpellier \\ Cedex 5, France
}

Submitted 12 October 2004: Accepted 11 April 2005

\begin{abstract}
Objective: To examine and analyse consumption changes over time of 24 food items between Sardinia and Malta.

Setting: The data were collected in 2001 in Sardinia and 2002 in Malta.

Design: A structured qualitative questionnaire, articulated around four main themes: food supply, transformation, preparation and consumption habits, was administered by face-to-face interviews with the help of a local person. It encompassed mainly open-ended questions, which allowed us to measure factors contributing to change. Subjects: Thirty mother-daughter pairs were interviewed in each insular territory.

Results: Despite a common trend revealing a shift away from cereals, pulses and potatoes to the benefit of meat products, fats and sugar, our results showed contrasting evolutions in food consumption between both insular societies. Fruit and vegetables, olive oil and fish, which are part of the main features of the Mediterranean diet, were among the top foods for which consumption frequency has increased in Sardinia. In Malta, besides an increase in olive oil and vegetable consumption, cheeses and desserts showed the highest increase. Along with modernity and improved living conditions, enhanced commercial availability and increased diversity of food preparation were also identified as factors contributing to food consumption changes.

Conclusions: Although the Sardo-Mediterranean model is evolving under the impact of modernisation, it is not disappearing. In Malta, however, modernity has led to a more sudden shift from a state of food shortage to one of affluence, but in a cultural context where the identity is no longer Mediterranean but Anglo-Saxon.
\end{abstract}

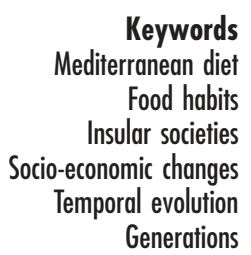

Despite the current trend towards globalisation of dietary habits due to the increasing supremacy of market over subsistence economy ${ }^{1}$, the evolution of food habits still leaves some room for social, spatial and cultural diversity. Therefore, food habits, which encompass food supply, transformation, preparation and consumption systems ${ }^{2}$, still deserve to be updated in order to be characterised. It is recognised that different food habits expose people to different health risks, acting either to promote or degrade health according to their spatial distribution in the population ${ }^{3}$. The link between health and the Mediterranean diet took form in many studies, the first of which, Ancel Keys' famous one, started in the 1950s and continued for over 30 years ${ }^{4}$. Later, other reviews also showed the health benefits of the Mediterranean dietary model, which can protect against cardiovascular diseases and some cancers and thus reduce the number of premature deaths linked to detrimental food habits ${ }^{5-9}$. The traditional Mediterranean diet is characterised by a high consumption of cereals and diverse pulses, fruit and vegetables. Meat consumption is low but fish is often consumed. There is virtually no milk nor butter but some cheeses and yoghurts. Olive oil is the only added lipid and wine is consumed regularly with main meals ${ }^{10}$.

The virtues of the Mediterranean dietary model are currently advocated as an 'art of living' and as a means of prevention against chronic degenerative diseases. Thus, defining what these lifestyles all around the Mediterranean Sea consist in is important, and is all the more necessary since indicators show that the Mediterranean diet is tending to disappear ${ }^{11,12}$. Therefore the actual characteristics of these lifestyles in terms of food habits need to be identified together with factors contributing to change.

The present study is original as it aimed to observe food habits and factors contributing to their change in two insular societies situated at the heart of the Mediterranean Sea - Sardinia and Malta - and compare them in terms of temporal evolution and spatial distribution. No research has previously been conducted in this area so far.

Because of reduced size and isolation due to naturally defined borders, insular environments seemed appropriate for bringing to light food habits preserved from 
external influences, a priori less numerous than on a continent. However, isolation, geographical conditions and historical events have not had the same impact on the evolution of Sardinian and Maltese societies. Indeed, until the second half of the 20th century, among all Mediterranean islands, Sardinia had remained the least exposed to other cultural influences and exchanges. Despite having been dominated by various invaders for at least two centuries, Sardinia has managed to preserve its original culture more intensively ${ }^{13}$ than Malta. Given its strategic geographic position, Malta was integrated, as early as the 16th century, into the trade navigation route of the central Mediterranean Sea, resulting in profound repercussions on the evolution of its culture ${ }^{14,15}$. Moreover, Maltese behaviours modelled themselves through contact with different foreign occupations; the last - by the British - had a tremendous impact on many aspects of society, including food habits. Therefore we thought that Sardinia would make an adequate candidate for bringing to light genuine Mediterranean food habits, and that the combination of Sardinia and Malta would make a suitable approach for unravelling the determinants of stable and changed food habits.

In the present paper, we show the evolution in time of the consumption of 24 food items in Sardinia and Malta. The paper also examines the factors determining food consumption change, which were measured by both bibliographical and field work.

\section{Subjects and methods}

\section{Analysis of Sardinian and Maltese socio-economic and cultural contexts and their evolution}

In order to study food habits as a whole, a preliminary analysis of the cultural and socio-economic context, as well as its evolution over the last century, was carried out because the interdependence between this field and food habits is great. Bibliographical and statistical data were collected and analysed to understand the historical facts and socio-economic developments that have shaped both societies and have been determinant in their evolution. To name but a few, we collected data on cultural identity, the British colonial presence (for Malta only), migration movements and urbanisation, as well as on the economy, with a focus on tourism and agriculture.

\section{Subjects}

To study food habit changes, inquiries were carried out over two generations - mothers and their daughters since women still have the monopoly of household tasks and play a key role in the transmission of food habits. The fieldwork took place at three different sites (i.e. urban, tourist and traditional). For each insular territory, it was planned to interview 10 mother-daughter pairs per inquiry site, that is to say 60 people in total. Recruitment of women was done in collaboration with general practitioners, or through parish play groups where mothers came with their young children, or with the use of a network. The direct approach of the latter method allowed us to reduce the number of refusals, and therefore participation bias. Fieldwork was carried out over 3 months in Sardinia (April, July and September 2001) and only 1 month in Malta (May 2002) due to our previously acquired experience in Sardinia and to the smaller size of the Maltese archipelago, making contacts and travelling easier.

\section{Questionnaire}

In both Sardinia and Malta, a structured qualitative questionnaire articulated around four main themes: food supply, transformation, preparation and consumption, was used. It encompassed mainly open-ended questions, which allowed in-depth investigation of food habits and factors contributing to their change. Evolution of consumption frequency was studied for 24 foods, representing the range of the different food groups (Cereals: bread, pasta; Fruit and vegetables: soup, pulses, fruit, vegetables, potatoes; Meat: beef, chicken, rabbit, pork, delicatessen, liver; Fish: dried cod and fish; Dairy products: milk, soft cheeses, hard cheeses; Fat: olive oil, seed oils, lard; Sweets: sugar, desserts; Alcoholic drinks: wine). Subjects were asked to compare (less, more or the same) their current food consumption (i.e. in the present family home) with that of their childhood (i.e. in the parental home).

The questionnaire was administered by face-to-face interviews with the help of a local person who translated Italian (or Sardinian) exchanges into French or English, and Maltese exchanges into English. Local translators had previously been thoroughly briefed on the study.

\section{Data analysis}

The social sciences adapted software, Le SPHINX Lexica 4.0 (Le Sphinx Développement, Chavanod, France), allowed us to semi-quantify qualitative data by performing an inventory of the range of variable modalities answered for each question. These steps were carried out ensuring that the qualitative aspect of the data was not lost in the computerisation process. Chi-square tests were performed for spatial comparison of the qualitative variables between the two islands using SPSS 10.1 (SPSS Inc., Chicago, IL, USA). Generational differences were analysed by performing Stuart-Maxwell tests, which enable comparisons of matching datasets, using STATA 7.0 (StataCorp, College Station, TX, USA).

\section{Results}

\section{Socio-economic characteristics of Sardinian and Maltese samples}

The socio-economic characteristics of the women are presented in Tables 1, 2 and 3 according to each generation and each study site within Sardinia and Malta. 
Table 1 Mean age of women according to generation and locality in Sardinia and Malta

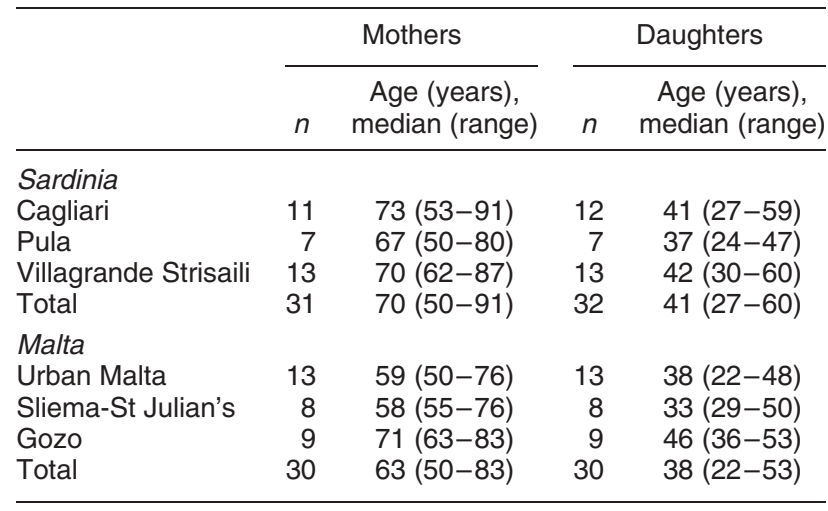

In total, 63 women were interviewed in Sardinia and 60 in Malta. The odd number of women in Sardinia is explained by the fact that in one case, one woman being both mother and daughter, three generations were interviewed.

The mean age of mothers was 70 years for Sardinians and 63 years for Maltese, whereas for the sample of daughters it was 41 years for Sardinians and 38 years for Maltese (Table 1). In both countries, a higher proportion of women of the 'daughter' generation lived with children (63\% vs. 39\% in Sardinia and 97\% vs. 33\% in Malta) rather than alone or with their spouse (Table 2). A majority of women of the 'mother' generation did not work outside the home (74\% of Sardinans and $83 \%$ of Maltese) whereas this was the case for only $44 \%$ of the Sardinian daughters and $50 \%$ of the Maltese daughters (Table 3).

\section{Temporal evolution of food consumption}

Temporal evolution of consumption for the 24 food items mentioned above is presented according to generation for both Sardinia and Malta as a whole in Figs 1 and 2.

Overall, the changed consumption of pulses, sugar, rabbit, seed oils, fish and beef contrasted Malta with Sardinia. In Sardinia, the decreased consumption of pulses is all the more important since it occurred over both the mothers' and daughters' generation. In Malta, although the consumption of pulses increased for both generations, it was to a lesser extent for the daughters. Moreover, baked

Table 2 Distribution of the proportion of women living with children between generations and localities, $n(\%)$

\begin{tabular}{lrc}
\hline & Mothers & Daughters \\
\hline Sardinia & & \\
Cagliari & $3(27)$ & $5(42)$ \\
Pula & $2(29)$ & $6(86)$ \\
Villagrande Strisaili & $7(54)$ & $9(69)$ \\
Total & $12(39)$ & $20(63)$ \\
Malta & & \\
Urban Malta & & $12(92)$ \\
Sliema-St Julian's & $8(62)$ & $8(100)$ \\
Gozo & $1(12)$ & $9(100)$ \\
Total & $1(11)$ & $29(97)$ \\
\hline
\end{tabular}

Table 3 Distribution of the proportion of women who have never worked outside the home between generations and localities, $n(\%)$

\begin{tabular}{lcr}
\hline & Mothers & Daughters \\
\hline Sardinia & & \\
Cagliari & $7(64)$ & $7(58)$ \\
Pula & $6(86)$ & $5(71)$ \\
Villagrande Strisaili & $10(77)$ & $2(15)$ \\
Total & $23(74)$ & $14(44)$ \\
Malta & & \\
Urban Malta & $10(77)$ & $8(62)$ \\
Sliema-St Julian's & $6(75)$ & $3(38)$ \\
Gozo & $9(100)$ & $4(44)$ \\
Total & $25(83)$ & $15(50)$ \\
\hline
\end{tabular}

beans were declared to be consumed by 40 (out of 60 ) Maltese women. Although these beans belong to the pulses group, the way they were reported to be prepared and consumed, the English breakfast way (with bacon, eggs and sausages; toasted with cheese; etc.), does not allow us to integrate them into the Mediterranean dietary model but rather into the British Anglo-Saxon one.

Vegetables and chicken were the two main food items, common to both countries and generations, for which consumption increased the most, whereas lard was at the top of the food items for which consumption decreased most compared with the past. Both nationalities of women declared that olive oil and/or seed oils, which were lacking in the past, have now replaced lard. It can be observed that a higher proportion of Maltese daughters than mothers decreased their consumption of seed oils, and that they are also slightly more numerous in having increased their olive oil consumption.

With the exception of chicken and processed cold meats, all other types of meat (beef, rabbit, pork and liver) underwent a decrease in consumption in Malta between the two generations; whereas in Sardinia, this holds true for pork and rabbit only. Although a decrease in beef consumption has been initiated in the second generation in both locations, it is to a lesser extent in Sardinia as this is the case for only half (i.e. 15) of the daughters sample. Fish consumption increased for both generations in Sardinia, but only for the mothers' generation in Malta since fish consumption decreased for the daughters' generation.

\section{Factors contributing to change}

Enhanced commercial availability

For the majority of both Sardinian and Maltese women, increases in fruit, vegetables and pulses consumption were attributed to greater availability and a wider range of these food products. In both samples, most of the older women declared that fruit and vegetables consumption used to be essentially limited to local production in the past and was also determined by seasonal rhythm.

Fish and sea products consumption were also reported to have increased due to greater availability and choice. 

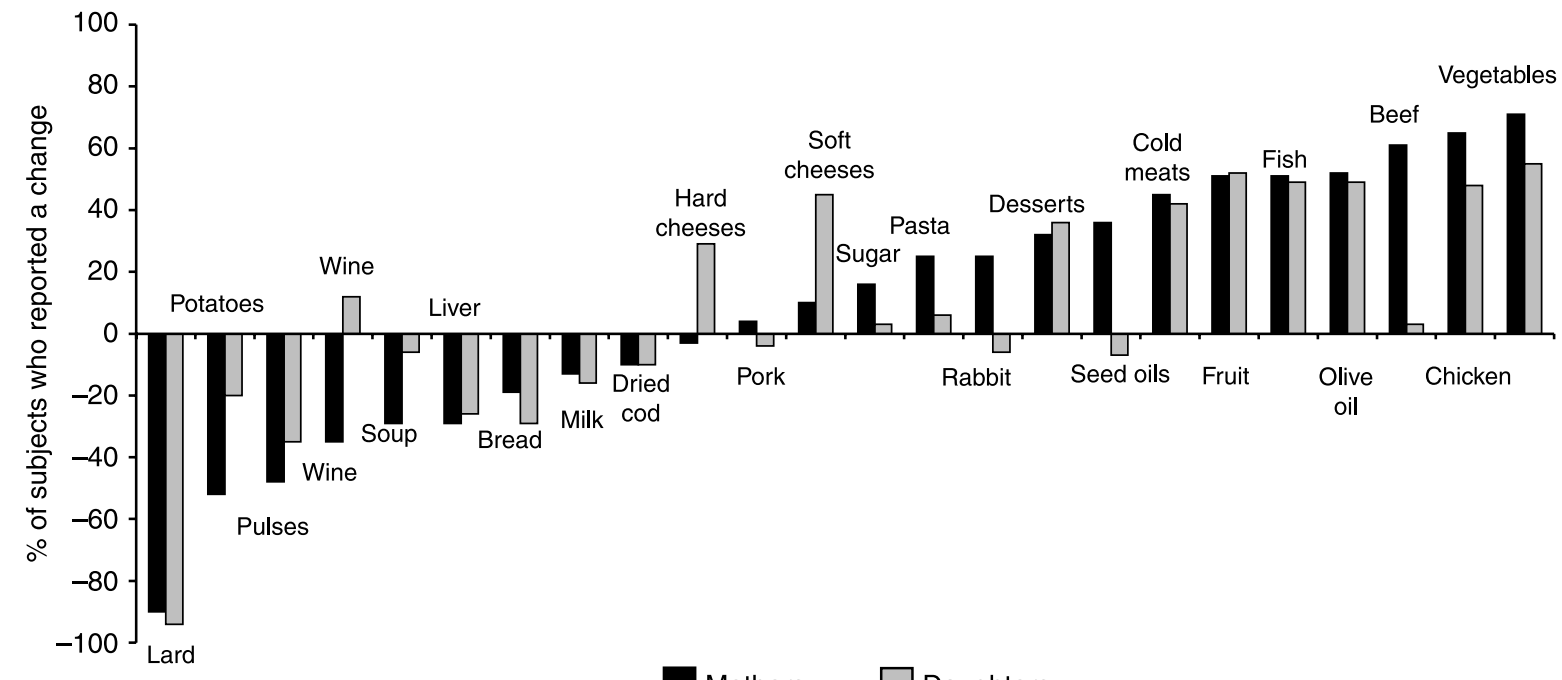

Mothers

Daughters

Fig. 1 Evolution of the consumption of the 24 food items in Sardinia according to each generation (balance $+/-$ )

In the past, because of lack of accessibility due to poor roads and connections with the rest of the island, hardly any women from the Sardinian traditional study site (Centre-East of Sardinia) used to eat any fish. As for dried cod, known to be traditionally consumed in Mediterranean countries, none of these women used to consume it before and they all have presently persisted in this habit of not eating any. Nowadays, fish and other sea products are made available locally thanks to an itinerant dealer who comes a couple of days a week to this remote agropastoral mountain village. Therefore, the opposite trend observed in the evolution of fish consumption between the two islands, in favour of Sardinia, is due to the traditional Sardinian sample having increased its fish consumption over the last decades.
Enhanced purchasing power was also reported to have favoured the increase in fish consumption among Maltese mothers. This generation also reported the end of fuel rationing - thus allowing fishing boats to go off shore which was a determining factor in the increase of fish consumption at adulthood. The second Maltese generation showed an aversion towards this food, increased by the difficulty in finding it fresh in the shops, which explains the current decrease in fish consumption.

In both countries, most women reported that cheese consumption increased due to a wider choice available than in the past. However, the increase was significantly more important in Malta than Sardinia $(+49$ and +11 respectively, $P<0.01)$. The famous 'Cheddar' was mentioned by $73 \%$ (44) of Maltese women. Grated cheese,

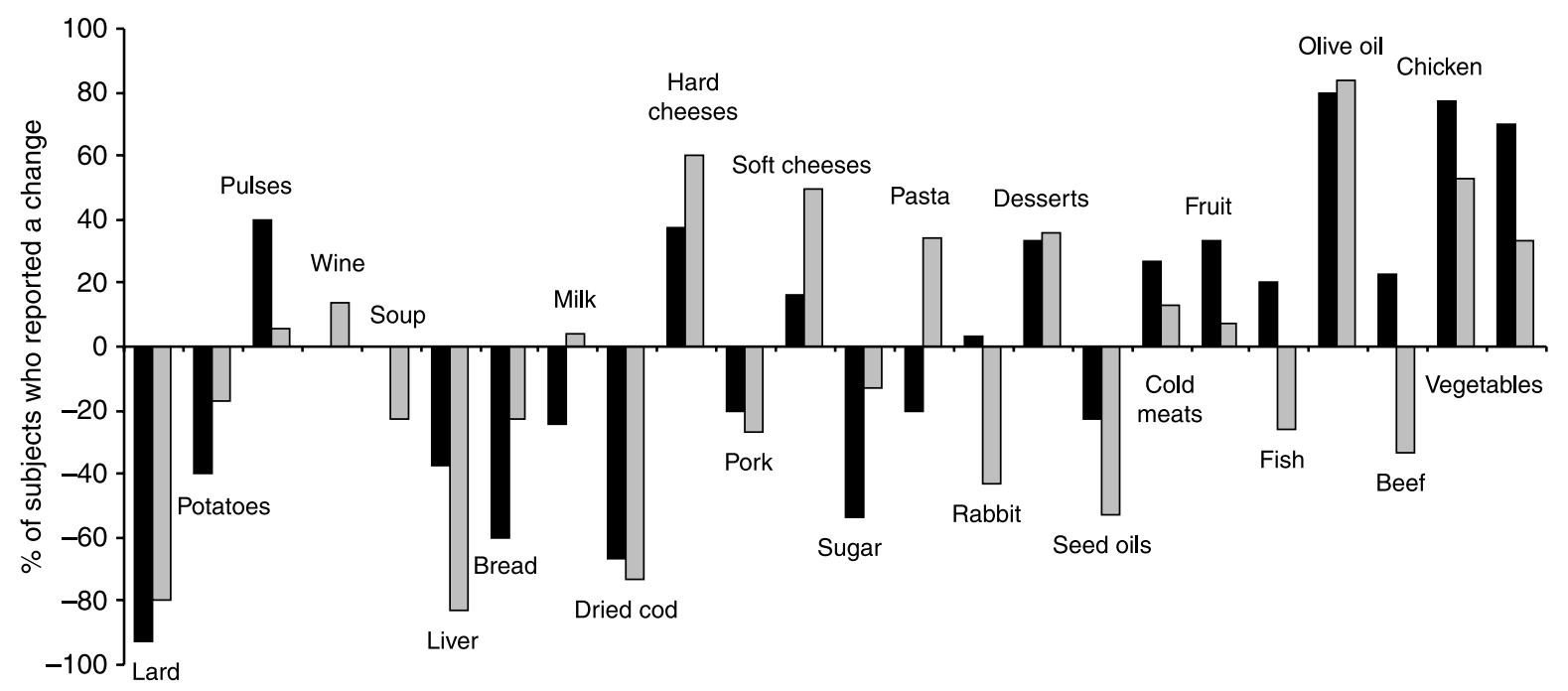

Mothers

Daughters

Fig. 2 Evolution of the consumption of the 24 food items in Malta according to each generation (balance $+/-$ ) 
Edam, Emmenthal and processed cheese are among the other hard cheeses most cited by Maltese women. In Sardinia, cheese consumption also increased, but Western types of cheeses were mentioned to be consumed by only six of 63 women in Sardinia versus 53 of 60 women in Malta and did not include cheddar at all. Instead, locally produced goat and sheep cheeses (e.g. Fiore Sardo, Pecorino Romano and Dolce Sardo) were preferentially consumed owing to the long-standing pastoral Sardinian tradition.

Both Sardinian and Maltese women from the first studied generation declared to have increased their beef consumption when purchasing power and availability became greater but also when ration tickets came to an end in Malta. For nine of 16 Maltese daughters, one of the main reasons evoked for eating less beef was the increased availability of other types of meat (e.g. poultry), fish and vegetarian surrogates (e.g. soya mince); this could also be attributed to an impact made by Maltese public health campaigns aimed at reducing red meat consumption. In Sardinia, this decline was rather attributable to fears about bovine spongiform encephalopathy for six of 18 women.

\section{Increased pace of life, enhanced variety and availability} of housebold appliances

The increase in consumption of cold meats has been progressing for two generations in both countries. Although not significantly different, the increase was greater in Sardinia $(+42)$ than in Malta $(+20)$. In Sardinia, increased purchasing power (for nine of 35 women), the increased pace of life due notably to women's remunerated activity (for eight of 35 women) and enhanced variety (for six of 35 women) and availability of household appliances (for two of 35 women), allowing for easier conservation, were declared as determinants that have contributed to this increase.

\section{Women's increased activity in the workforce}

In Sardinia, the decline in the consumption of pulses was directly linked to a decrease in consumption of the traditional minestrone soup for 11 out of 37 women. Up until a few years ago, due to poor conditions of living, minestrone soup was reported to be the staple food and therefore was consumed on a daily basis. Nowadays, not only is minestrone soup consumed less but it is also prepared in a different way, especially so among the daughters' generation (although not significantly different, $65 \%$ vs. $46 \%$ ); as the number of younger women going to work increases, the amount of time they dedicate to cooking decreases. Whereas minestrone soup was in the past essentially made from potatoes and pulses rather than fresh vegetables, today, for convenience, five Sardinian women out of 35 declared becoming more and more accustomed to using readyto-use frozen vegetables for soup which are currently available in supermarkets.
In Malta, significantly more women from the first than from the second generation currently prepared soups in the same way their mothers used to $(63 \%$ vs. $37 \%$; $P<0.05)$. It was reported that the way soup preparation has changed nowadays compared with the past is that it contains one type of vegetable only, thus reducing diversity in ingredients, which is central to the Mediterranean dietary model.

\section{Historical and cultural determinants}

\section{Migration}

For Malta, bibliographical analysis revealed that not only has the Anglo-Saxon influence been ongoing for over a century and a half (through colonial presence), but also that it has grown stronger through migration to and from Anglo-Saxon countries (including Great Britain, the USA, Canada and Australia) which occurred throughout the 20th century in order to escape precarious living conditions. This constitutes a major difference with Sardinia because not only did very few Sardinians, compared with Maltese, leave their country, but for those who did so, they remained mostly in the Mediterranean area. Maltese emigration was at its highest between 1946 and 1970 and started to reverse back from 1975 onwards. Due to high rates of unemployment in the host countries, Maltese migrants started to turn back en masse at the end of the 1980s until the beginning of the 1990s. Therefore, culturally impregnated by the host society's lifestyle, returning migrants brought back to Malta food habits that differed from local ones at that period of time.

\section{Tourism}

Further analysis showed that British cultural influence continued after Independence in 1964 with the development of international mass tourism, of which $60 \%$ were British tourists in the 1980s. Although in decline, the British are still predominant and currently represent $41 \%$ of tourist arrivals. As a result, until recently, Maltese food was totally absent from restaurant menus. Instead, restaurant owners used to serve British food such as prawn cocktail, fish and chips, etc.

This is another essential difference with Sardinia where, on the one hand, tourism developed later and, on the other hand, it has still not reached proportions of mass tourism due to, among other things, the greater size of the island. Moreover, foreign tourists represent only $21.4 \%$ of tourist arrivals, among which the French and Spanish are the most represented.

\section{Discussion}

The design of the study allowed us to show a contrast in the evolution of food consumption and habits over time not only between Sardinia and Malta, but also within each 
island as well according to generation. Interviewing two generations was a deliberate choice as they personify two important historical moments (the Second World War for the mothers' generation and the 1970s for the daughters) which represent two opposite situations and therefore two different lifestyles. While the post-war period was marked by food shortages, the 1970s marked the beginning of improvement for living standards. The changes generated by socio-economic developments, such as migration, tourism and enhanced commercial availability amongst other things, were expected to lead to an evolution in food habits from one generation to the next.

Other studies have reported some similar trends in the food consumption of both Sardinian and Maltese. Our results regarding increased fish consumption are in agreement with a recent survey carried out in Sardinia confirming that sea products, and especially crayfish and fried squid, were as much appreciated as roast chicken by students of Cagliari and were even preferred to hamburgers. Moreover, this survey highlighted the fact that Sardinians are the foremost sea bream consumers in the whole of Italy. On average, a Sardinian household buys about $2.5 \mathrm{~kg}$ of sea bream per year whereas Northern Italians only buy $900 \mathrm{~g}^{16}$.

The important consumption of cheese found in Malta is confirmed by Rolland ${ }^{17}$ who reported that, in Malta, cheese is the most imported food among dairy products (67\% of the total value) among which 'vache qui rit' is the leader of cheese spreads. Moreover, this report also confirmed the growing success of such food products in Malta, which can be attributed to a large consumption of sandwiches, another Anglo-Saxon influence. Cheese consumption is highly conditioned by international imports, mainly from Anglo-Saxon and Northern European countries (e.g. United Kingdom, Australia, Denmark and France).

In Sardinia and Malta alike, these changes in food consumption reflect the crucial role that modernity has played. These results show that, over the last decades, modernity has allowed an improvement of diet, through greater diversity and generalised access to all food groups by the whole population spectrum. Indeed, in both Sardinian and Maltese societies, at the beginning of the 20 th century, it is reported that bread played a central role in the diet ${ }^{18-20}$. Counihan ${ }^{18}$ also showed that even though bread still holds an important place in the current diet, its place has decreased due to increased availability of other foods. Despite the undeniable role of the latter improvement in quality of life (especially so among the sample of women belonging to the first generation) and longevity, modernity is also a bearer of bad eating habits by promoting excessive consumption of meat and foods rich in fats (e.g. cheeses) and sugar (e.g. dessert), which had been sorely lacking during the Second World War. One can thus witness two kinds of changes, those which are beneficial to health and those which are detrimental as they can, directly or indirectly, be held responsible for the development of some pathologies. In the case of Sardinia, modernity has heightened the traditional Sardo-Mediterranean dietary model for both generations (i.e. increases in fruit and vegetables, olive oil and fish consumption). Although the consumption of cheese was shown to be on the increase, our study shows that Sardinians still favour locally produced goat and sheep cheeses, sources of $\alpha$-linolenic acid which protect against cardiovascular diseases $^{6}$. But we also highlighted that Sardinia seems to be losing the benefits of a high consumption of pulses which it knew at earlier generations. In Malta, the outcome is more of a mixed blessing. Although modernity, along with public health recommendations, has also favoured changes, those that tend towards healthier food practices (e.g. vegetables) come with some which are detrimental to health (e.g. cheeses and desserts). Moreover, we highlighted on several occasions that Maltese did not display Mediterranean food habits but rather Anglo-Saxon ones. These include, among others, lack of diversity (e.g. soup preparation), consumption of pulses of which baked beans form an important part and are usually prepared the British way, and consumption of cheese with cheddar (containing 33\% fat of which 18\% are saturated) being part and parcel of the Maltese sample's food habits. Regarding the decreased fish consumption among the second generation of Maltese women, it is difficult to find a fresh supply of fish at supermarkets and hence most sales occur at fishmongers, which are not homogeneously displayed on the archipelago but localised in the main urban and tourist centres. Unfortunately, this does not encourage regular consumption among the Maltese population who seems to turn towards transformed fish products (e.g. fish fingers) instead, often rich in saturated fatty acids and salt.

What is important in our findings is that although both societies have undergone changes in their food habits, in Sardinia food changes occurred within the frame of the Mediterranean food culture, whereas in Malta we showed that the Anglo-Saxon cultural context due to past colonial presence, migration and tourism has had a deep impact on food habit changes. Thus it can be concluded from these results that changes in each society have not had the same impact on the evolution of food practices. It has reinforced the Sardo-Mediterranean cultural model in Sardinia and the Anglo-Saxon one in Malta.

This study presents a few limitations. First, we are aware that declared data may not correctly reflect real changes since declarations can convey change as much as an aspiration to change; however, these results did allow us to measure what constitutes the norm regarding lifestyles or nutritional recommendations ${ }^{21}$. Second, for some variables, the statistical power was low because of the small sample size. Nevertheless, the design of the study based on investigations over two generations proved to be efficient in measuring the existence and the extent of change in both insular societies. Moreover, the conditions 
under which this study was done were the most favourable for eliminating confounding factors which could have arisen from the study of two separated generations. The design of our study therefore contributes to increase the statistical value of our findings.

This is a pioneering study in the field of food habits in insular societies and has provided precious and useful information. Indeed, these findings could be the basis of nutritional recommendations to implement a real Mediterranean diet in Malta. Moreover, they could be used as a model for other past and current British colonies (e.g. Cyprus and Gibraltar) which have also lost the Mediterranean dietary model.

\section{Acknowledgements}

This work would not have been possible without the financial support of the 'Ligue Contre le Cancer' (grant given by the Hérault and Languedoc-Roussillon Committees) and the Regional Council of Languedoc-Roussillon in the form of a PhD studentship awarded to S.T.

\section{References}

1 Aubaile-Sallenave F. Une image moderne de la cuisine méditerranéenne In: Alimentation et nourritures autour de la méditerranée. Paris: Editions Karthala et Ciheam, 2000.

2 Calvo E. La diversité des pratiques alimentaires dans le macro-espace social. In: Alimentation et régions. Actes du colloque 'Cuisines, régimes alimentaires, espaces régionaux', Nancy, France, 24-27 septembre 1987. Nancy: Presses Universitaires de Nancy, 1989.

3 Kromhout D. Food consumption patterns in the Seven Countries Study. Seven Countries Study Research Group. Annals of Medicine 1989; 21(3): 237-8.

4 Keys A, Menotti A, Karvonen MJ, Aravanis C, Blackburn H, Buzina R, et al. The diet and 15-year death rate in the Seven Countries Study. American Journal of Epidemiology 1986; 124: $903-15$.

5 Ferro-Luzzi A, Branca F. Mediterranean diet, Italian-style: prototype of a healthy diet. American Journal of Clinical Nutrition 1995; 61(Suppl.): 1338S-45S.
6 Kushi LH, Lenart E, Willett W. Health implications of Mediterranean diet in light of contemporary knowledge. 1. Plant foods and dairy products. American Journal of Clinical Nutrition 1995; 61(Suppl.): 1407S-15S.

7 Kushi LH, Lenart E, Willett W. Health implications of Mediterranean diet in light of contemporary knowledge. 2. Meat, wine, fats and oils. American Journal of Clinical Nutrition 1995; 61(Suppl.): 1416S-27S.

8 Corpet DE, Gerber M. Alimentation Méditerranéenne et Santé. I. Caractéristiques. Maladies cardio-vasculaires et autres affections. Médecine et Nutrition 1997; 4: 129-42.

9 Gerber M, Corpet DE. Alimentation Méditerranéenne et Santé. II. Cancers. Médecine et Nutrition 1997; 4: 143-54.

10 Ferro-Luzzi A, Sette S. The Mediterranean diet: an attempt to define its present and past composition. European Journal of Clinical Nutrition 1989; 43(Suppl. 2): 13-29.

11 Scali J, Richard A, Gerber G. Diet profiles in a population sample from Mediterranean southern France. Public Health Nutrition 2000; 4(2): 173-82.

12 Ferro-Luzzi A, James WP, Kafatos A. The high-fat Greek diet: a recipe for all? European Journal of Clinical Nutrition 2002; 56(9): 796-809.

13 Berlinguer L, Mattone A. La Sardegna. Storia d'Italia. Le regioni dall'Unita a oggi. Torino: Einaudi, 1998.

14 Cassar C. Society, Culture and Identity in Early Modern Malta. Malta: Mireva Publications, 2000.

15 Cassar C. A Concise History of Malta. Malta: Mireva Publications, 2000.

16 Isola sarda newsletter [online], November 2001. Available at http://www.isolasarda.com/newsletter2001.htm\#NOV EMBRE\%202001

17 Rolland G. Le marché des produits laitiers à Malte. Fiche de synthèse. Missions économiques. Location: Ambassade de France à Malte, 2002. Also available at http://www.dree.org/ malte/

18 Counihan C. Bread as world. Food habits and social relations in modernizing Sardinia. In: The Anthropology of Food and Body. Gender, Meaning and Power. New York: Routledge, 1999.

19 Cassar C. Everyday life in Malta in the nineteenth and twentieth centuries. In: The British Colonial Experience 1800-1964. The Impact on Maltese Society. Malta: Mireva Publications, 1988.

20 Cassar C. Fenkata: An Emblem of Maltese Peasant Resistance? Malta: Ministry for Youth and the Arts, 1994.

21 Poulain JP. Manger aujourd'bui: attitudes, normes et pratiques. Paris: Editions Privat, 2002. 